\title{
Otoplasty for prominent ears - demographics and surgical timing in different populations
}

Kajosaari, Lauri

2017-09

Kajosaari , L , Pennanen , J \& Klockars , T 2017 , ' Otoplasty for prominent ears -

demographics and surgical timing in different populations ' , International Journal of Pediatric

Otorhinolaryngology, vol. 100 , pp. 52-56 . https://doi.org/10.1016/j.jporl.2017.06.014

http://hdl.handle.net/10138/297949

https://doi.org/10.1016/j.ijporl.2017.06.014

publishedVersion

Downloaded from Helda, University of Helsinki institutional repository.

This is an electronic reprint of the original article.

This reprint may differ from the original in pagination and typographic detail.

Please cite the original version. 


\title{
Otoplasty for prominent ears - demographics and surgical timing in different populations
}

\author{
Lauri Kajosaari*, Juhani Pennanen, Tuomas Klockars \\ Department of Otorhinolaryngology - Head and Neck Surgery, Helsinki University Hospital and University of Helsinki, Helsinki, Finland
}

\section{A R T I C L E I N F O}

\section{Article history:}

Received 30 March 2017

Received in revised form

14 June 2017

Accepted 14 June 2017

Available online 16 June 2017

\section{Keywords:}

Prominent ears

Otoplasty

Surgery

\begin{abstract}
A B S T R A C T
Introduction: Prominent ears are seen in approximately 5\% of the population. This benign condition can be treated surgically to reduce or prevent psychological and social problems, most commonly in children before the start of school. Our aim was to examine the demographic characteristics of patients undergoing prominent ear surgery in Finland, and compare findings with international data.

Methods: A retrospective study of all the patients treated for prominent ears in our academic tertiary care referral center during 2007-2011 was performed to gather demographic details of operated patients. A systematic review of published series of prominent ear surgery after the year 2000 was performed to gather demographic details for international comparison.

Results: A total of 180 patients were operated in our institution for prominent ears, most of the cases (78.9\%) were bilateral. Age at operation ranged between 3 and 36 years, with mean of $9.2 \mathrm{y}$ and median of $7 \mathrm{y}$. The most common reason for seeking operative treatment was aesthetic complaint, followed by bullying.

Review process gathered 20 publications, describing a total of 4433 patients who had been operated for prominent ears. There was wide variation the mean age at operation, ranging 7-38 y while the mean overall was $15.0 \mathrm{y}$. Gender distribution of patient samples was also very variable, with percentage of females ranging from 38 to $71 \%$ (overall 52\%). There was also considerable variation in the tendency to perform unilateral operations: from $0 \%$ up to $21 \%$ of the reported population. There were no statistically significant correlations linking these demographic variables, though there was a trend that females are more likely to have this operation performed at an older age.

Conclusions: The treatment culture of prominent ear surgery varies substantially in international comparison. The age at which this operation is performed showed most variation. Omitting some of the basic demographic variables while reporting the results of surgery was common in the reviewed publications.
\end{abstract}

(c) 2017 Published by Elsevier Ireland Ltd.

\section{Introduction}

The diagnosis of prominent ears is always subjective. Although extensive measurements of auricular dimensions and angles are available, the definition of "normal" or "prominent" is obscure. The prevalence of prominent ears has suggested to be approximately $5 \%$ [12]. Otoplasty is one of the most common pediatric otorhinolaryngological operations. Indications are mainly aesthetic although functional problems are also possible. Corrective procedures are performed in a wide variety of settings: in private and public clinics,

\footnotetext{
* Corresponding author. Department of Otorhinolaryngology and Head \& Neck Surgery, Helsinki University Hospital, P.O.Box 220, FI-00029 HUH, Helsinki, Finland. E-mail address: lauri.kajosaari@hus.fi (L. Kajosaari).
}

by plastic surgeons and otolaryngologists, to pediatric and adult patients [16]. (see Figs. 1 and 2).

Surgical correction of prominent ears is usually done from approximately age 4-5 onwards, when the size of auricle approaches adult dimensions [1] and good compliance in the postoperative period can be expected [8]. Timing the surgery before the start of school is a common goal in order to prevent incidents of bullying [12], but significant proportion of operations are done also in the adulthood. The scientific literature regarding prominent ear surgery has been mainly focused on description of specific variants in the surgical techniques, reflected by over 200 published technical variants [12].

We wanted to study the demographics of otoplasty based on published patient series in different populations. A patient series from our own department was gathered for comparison. 


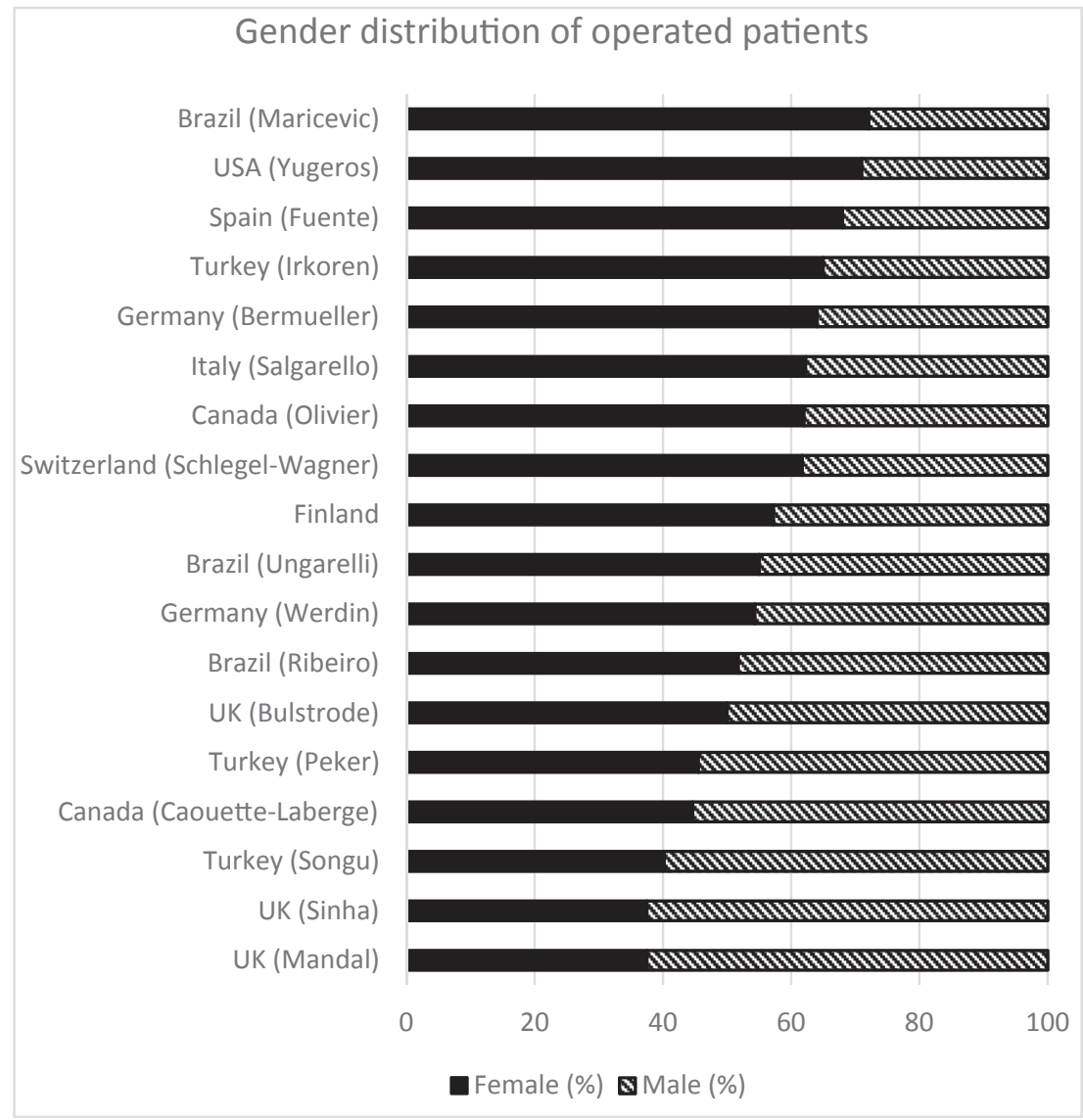

Fig. 1. Gender distribution of patients operated for prominent ears in international comparison.

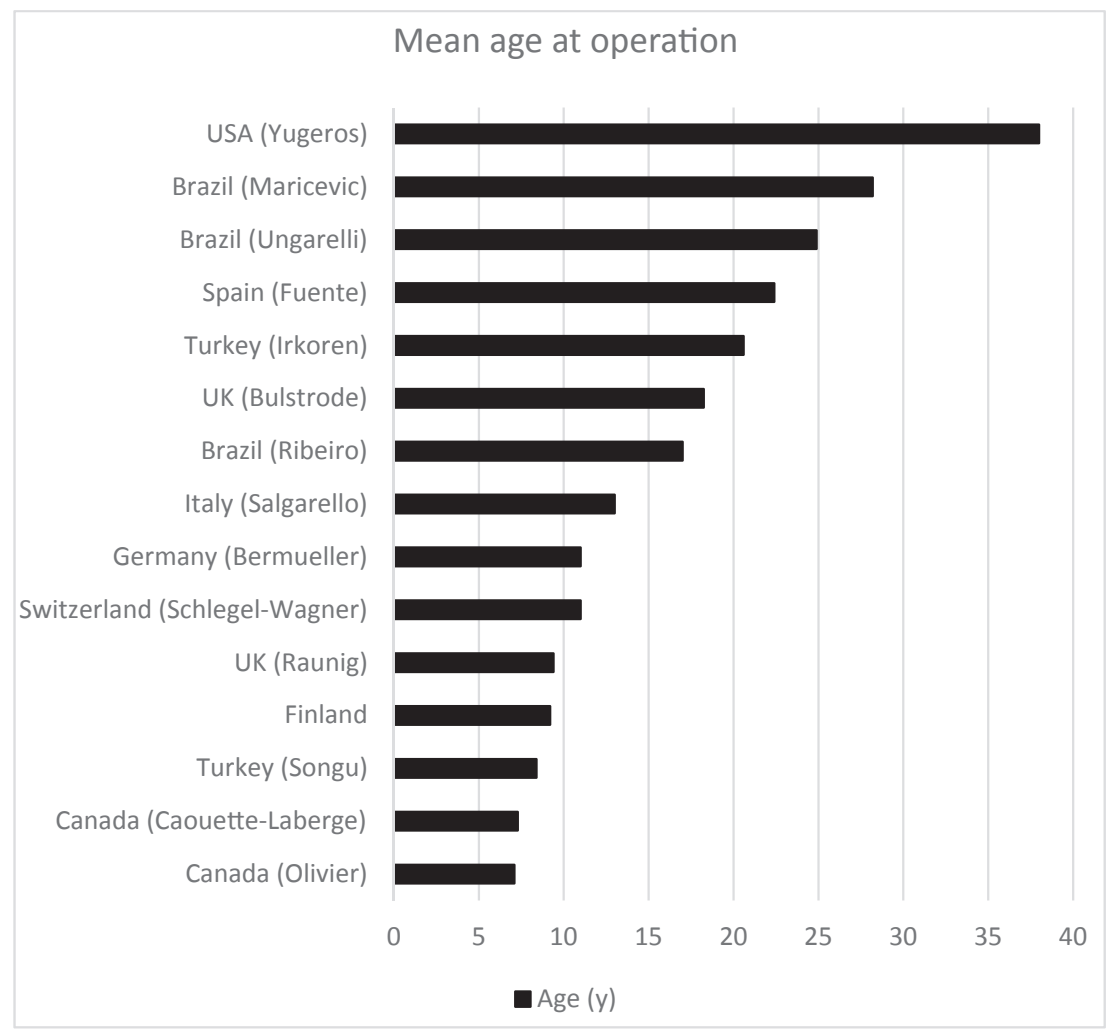

Fig. 2. Mean age of patients operated for prominent ears in international comparison. Publications with age limits in material omitted. 


\section{Patients and methods}

\subsection{Data from our institution}

$\mathrm{HUH}$ is a tertiary care referral center attending specialist level health care to approximately 1.5 million people in Southern Finland. Electronic hospital records (covering years 2007-2011) were searched to identify all patients referred because of prominent ears to the Department of Otorhinolaryngology and Head \& Neck surgery. The identification was based on the ICD-10 code Q17.5. Data on patient demographics and surgical notes were gathered. The reason for referral was categorized as follows: (1) aesthetic dissatisfaction, (2) bullying, (3) functional reasons, (4) not specified. In addition, we collected data on gender, age at operation, unilateral or bilateral procedure, and primary or revision otoplasty.

\subsection{Literature search}

A literature search of the PubMed database for search term "otoplasty" was performed. The search was limited to publications in English and years 2000-2016. Abstracts were reviewed to identify publications with patient series of at least 100 patients. This review process gathered 20 original articles for demographic analyses.

The Statistical analysis was performed using the Statistical Program for Social Sciences (SPSS for Windows, Version 22.0 statistical software; SPSS Inc, Chicago, IL, USA). Correlations were analyzed by calculating Pearson correlation coefficients. P-values were all two-tailed and the significance level was set at 0.05 .

Institutional review board approval was obtained for this retrospective study based on hospital records only.

\section{Results}

We were able to identify 180 patients who had a surgical operation performed for uni- or bilateral prominent ears in our department (Table 1 ). The majority of patients were female (58\%), age at operation ranged from 3 to 36 years, with a mean of $9.2 \mathrm{y}$ and median of $7 \mathrm{y}$. The operation was bilateral in 142 cases (79\%).

The indication for surgery was clearly described in majority of

Table 1

Characteristics of patients operated for prominent ears in our institution.

\begin{tabular}{ll}
\hline Number of patients & 180 \\
\hline Total & $103(57.2 \%)$ \\
Female & $77(42.8 \%)$ \\
Male & \\
Age at operation (y) & \\
Mean & 9.2 \\
Median & 7 \\
Range & $3-36$ \\
& \\
Primary operations & $171(95 \%)$ \\
Revision operations & $9(5 \%)$ \\
Side of prominent ear operation & \\
Right & \\
Left & $17(9.4 \%)$ \\
Bilateral & $21(11.7 \%)$ \\
No reason for referral specified & $142(78.9 \%)$ \\
Reason(s) for referral specified & \\
Aesthetic complaint & $30(16.7 \%)$ \\
Bullying & $150(83.3 \%)$ \\
Functional problems & $94(62.7 \%$ of 150$)$ \\
\hline & $44(29.3 \%$ of 150$)$ \\
& $21(14 \%$ of 150$)$ \\
\hline
\end{tabular}

cases (150/180, 83\%). Aesthetic complaint was most common (94/ $150,63 \%)$, followed by bullying $(44 / 150,29 \%)$ and functional complaint (21/150,14\%). Functional complaints included painful twisting when sleeping, increased tendency for sunburn/frostbites and problems with headgear (hats, glasses, hearing aids etc.) For the subgroups with only one listed indication, aesthetics had a mean age of $8.9 \mathrm{y}$ (median $7 \mathrm{y}, \mathrm{n}=84$ ), bullying mean age of $8.7 \mathrm{y}$ (median $8 \mathrm{y}, \mathrm{n}=38$ ) and functional problems mean age of $13.0 \mathrm{y}$ (median $11 \mathrm{y}, \mathrm{n}=21$ ). Vast majority of the operations were primary otoplasties (171 cases, 95\%).

Our literature search revealed 20 original publications (English language, more than 100 patients) resulting a total of 4433 patients after our cohort is included (Table 2). The age at operation ranged from 2 to 66 years, with a mean of 15 years (excluding publications with age limits, $n=3011)$. The gender distribution was even $(52 \%$ female, $\mathrm{n}=3840$ ). However, the gender distribution in different publications ranged from 38 to $72 \%$ being females. The number of bilateral or unilateral procedures was reported for a total of 2249 patients, of those $92 \%$ were bilateral $(n=2432)$ and $8 \%$ unilateral $(\mathrm{n}=183)$. The percentage of bilateral cases in different publications varied from 79 to $100 \%$. The distribution of primary or revision operations in the published series were mostly omitted, and no meaningful overall average could be calculated. The rate of revision operation ranged from $0 \%$ to $33.8 \%$ for those reported (Table 2 ).

No statistically significant correlations were found between the demographic variables.

The gender distribution in a sample (percentage of females) correlated with mean age at operation by Pearson correlation coefficient $0.53(\mathrm{p}=0.063)$. The percentage of bilateral operations in a sample correlated with mean age at operation by Pearson correlation coefficient $0.22(\mathrm{p}=0.57)$. The gender distribution in a sample (percentage of females) correlated with percentage of bilateral operations by Pearson correlation coefficient 0.26 $(\mathrm{p}=0.43)$.

\section{Discussion}

The diagnosis of prominent ears is subjective. As aesthetic complaints are the most common indication for surgery, we expected variations also in the demographics of published patient series. The greatest variation was observed in the age at surgery. The mean age at operation was youngest in the two publications from Canada, approximately 7 years [6,15]. In addition to these two publications, only in series published from Turkey, United Kingdom and our series (Finland) had age average below 10 years [18,23]. The oldest patients were in a series from USA (mean 38 years) [27], and an age average older than 20 years was reported in two publications from Brazil [14,25], one from Spain [7] and one from Turkey [11].

Whether the variation in the age at surgery reflects true differences in different populations rather than publication can be discussed. There was no obvious bias in the reporting institutions, as both ends of the variation included plastic surgical and otorhinolaryngological units as well as university and private hospitals. It is possible, that in some countries, institutions operating more adults are more active in reporting their results. However, as almost all 20 publications also reported pediatric cases this seems to be an unlikely explanation. It seems reasonable to assume that variation in age at operation reflects true differences in operative cultures.

In countries with a strong funding for public sector hospitals, such as Finland, it can be expected that the results are more or less extrapolated to the country as a whole. However, the influence of privately funded clinics is greater in countries such as USA, leading to greater variation within a country. Aesthetic and cosmetic surgery is much more common in Brazil and USA than other countries 
Table 2

Publications of prominent ear surgery with at least 100 patients from 2000 to 2016 .

\begin{tabular}{|c|c|c|c|c|c|c|c|}
\hline Location (First author) & $\begin{array}{l}\text { Publication } \\
\text { year }\end{array}$ & $\begin{array}{l}\text { Sample } \\
\text { size }\end{array}$ & $\begin{array}{l}\text { Female - male } \\
(\%)\end{array}$ & $\begin{array}{l}\text { Age (mean, } \\
\text { y) }\end{array}$ & $\begin{array}{l}\text { Age (range, } \\
\text { y) }\end{array}$ & $\begin{array}{l}\text { Bilateral - unilateral } \\
(\%)\end{array}$ & $\begin{array}{l}\text { Revision operations included in published } \\
\text { series (\%) }\end{array}$ \\
\hline Canada (Caouette-Laberge L) [6] & 2000 & 500 & $45-55$ & 7.3 & $2-53$ & $95-5$ & 0 \\
\hline United States (Yugeros P) [27] & 2001 & 100 & $71-29$ & 38 & $4-66$ & $93-7$ & NA \\
\hline Turkey (Peker F) [17] & 2002 & 178 & $46-54$ & NA & $7-38$ & $93-7$ & NA \\
\hline $\begin{array}{l}\text { United Kingdom (Bulstrode NW) } \\
\text { [4] }\end{array}$ & 2003 & 114 & $50-50$ & 18.3 & $3-66$ & $88-12$ & NA \\
\hline United States (Burstein FD) [5] & 2003 & 100 & NA & NA & NA & $100-0$ & NA \\
\hline United States (Stucker FJ) [24] & 2003 & 329 & NA & NA & NA & NA & NA \\
\hline United Kingdom (Mandal A) [13] & 2006 & 203 & $38-62$ & 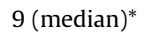 & $5-16^{*}$ & $100-0^{* *}$ & NA \\
\hline Germany (Werdin F) [26] & 2007 & 278 & $54-46$ & NA & $5-65$ & NA & NA \\
\hline Italy (Salgarello M) [20] & 2007 & 135 & $62-38$ & 13 & $4-34$ & $97-3$ & NA \\
\hline Canada (Olivier B) [15] & 2009 & 104 & $38-62$ & 7.1 & NA & $95-5$ & NA \\
\hline $\begin{array}{l}\text { Switzerland (Schlegel-Wagner } \\
\text { C) [21] }\end{array}$ & 2010 & 222 & $62-38$ & 11 & $3-31$ & NA & 0 \\
\hline Brazil (Maricevich P) [14] & 2011 & 111 & $72-28$ & 28.2 & $5-65$ & NA & NA \\
\hline Brazil (Ribeiro JAS) [19] & 2012 & 897 & $52-48$ & 17 & $5-65$ & NA & 33.8 \\
\hline Germany (Bermueller C) [2] & 2012 & 164 & $64-36$ & 11 & $5-42$ & NA & 12.2 \\
\hline United Kingdom (Sinha M) [22] & 2012 & 227 & $38-62$ & $\mathrm{NA}^{*}$ & $6-16^{*}$ & $92-8$ & NA \\
\hline Spain (de la Fuente A) [7] & 2012 & 100 & $68-32$ & 22.4 & NA & $96-4$ & NA \\
\hline Turkey (Songu M) [23] & 2014 & 107 & $40-60$ & $8.4^{*}$ & $4-16^{*}$ & $85-15$ & NA \\
\hline United Kingdom (Raunig H) [18] & 2014 & 164 & NA & 9.4 & $4-32$ & $82-18$ & NA \\
\hline Turkey (Irkoren S) [11] & 2014 & 100 & $65-35$ & 20.6 & $6-55$ & $90-10$ & NA \\
\hline Brazil (Ungarelli LF) [25] & 2016 & 120 & $55-45$ & 24.9 & NA & $100-0^{* *}$ & 0 \\
\hline Finland (Kajosaari L) & 2016 & 180 & $57-43$ & 9.2 & $3-36$ & $79-21$ & 5.0 \\
\hline
\end{tabular}

NA $=$ Not available.

*Adult patients excluded from the study.

** Unilateral cases excluded from the study.

in the review material [10]. Cosmetic surgery is constantly gaining popularity and reflects also trends in the economy [3]. In preschool children, the choice to pursue surgical treatment for prominent ears is highly dependent on parental opinion and more focuses on preventing possible future bullying, while with increasing age the decision is more individual and more guided by self-esteem/body image and possibly history of already realized bullying [9]. Thus the context and surroundings in which and individual receives prominent ear surgery can be very different.

Also gender distribution showed considerable variation, although in the pooled results for 3840 patients, the distribution was even with $52 \%$ of patients being female. Four publications broke the 65-35 female-male ratio: In two of these publications (USA, Brazil), more than $70 \%$ of operated patients were female $[14,27]$. The mean age of patients in these four were among the five publications with the highest mean age at operation. However, the correlation between the gender distribution and mean age at operation did not reach statistical significance (Pearson correlation $0.53 ; \mathrm{p}=0.063$ ). In the three publications that reported a male percentage over $60 \%$ two were limited only to pediatric cases (both from UK) [13,22] and the third had the lowest reported mean age of the entire review material (Canada) [15]. These results suggest that relatively more males receive prominent ear surgery at an earlier age compered to age groups older than 20 years where females are the majority.

Unilateral operations for prominent ears comprised $8 \%$ of patients overall, ranging from 0 to $21 \%$ in different populations. The tendency to perform unilateral procedures was most common in our institution (21\%) followed by the patient series from Turkey and UK, where unilateral procedures constituted at least $15 \%$ of the operations $[18,23]$. There are two possible explanations for the imbalance in the uni-vs bilateral operations: (1) It is possible, that unilateral prominence is more common in certain populations or (2) There is difference in the treatment culture regarding the operation. By operating both ears it might be easier to reach symmetric results. However, to our knowledge there are no studies on the patient satisfaction regarding uni- or bilateral operations. This would be important as operating both ears naturally increases the complication risks (keloid formation, postoperative infections and bleeding).

The specific techniques used in otoplasty showed considerable variation, as could be expected. Anatomic details and the surgeon's preference form the basis of decision making, influenced also by the treatment culture of the institution where surgery is performed. As numerous technical variants give comparable results, the field can be expected to remain heterogeneous in this matter. The rates of revision surgery or surgical complications were reported only in a fraction of articles included in Table 2, and thus omitted from our analysis.

\section{Conclusions}

The wide variation seen patients receiving surgery for prominent ears reflects differences in treatment culture between individual institutions as well as between countries, especially regarding age at operation.

\section{Conflicts of interest}

The authors report no conflicts of interest.

\section{Acknowledgements}

The project received financial support from the Stiftelsen Dorothea Olivia, Karl Walter och Jarl Walter Perkléns Minne, Finland.

\section{References}

[1] J.E. Adamson, C.E. Horton, H.H. Crawford, The growth pattern of the external ear, Plast. Reconstr. Surg. 36 (1965) 466-470.

[2] C. Bermueller, H. Kirsche, A. Sebert, N. Rotter, Quality of life and patients' satisfaction after otoplasty, Eur. Arch. Otorhinolaryngol. 269 (2012) 2423-2431.

[3] P.N. Broer, S.M. Levine, S. Juran, Plastic surgery: quo vadis? Current trends and future projections of aesthetic plastic surgical procedures in the United States, Plast. Reconstr. Surg. 133 (2014) 293e-302e. 
[4] N.W. Bulstrode, S. Huang, D.L. Martin, Otoplasty by percutaneous anterior scoring. Another twist to the story: a long-term study of 114 patients, Br. J. Plast. Surg. 56 (2003) 145-149.

[5] F.D. Burstein, Cartilage-sparing complete otoplasty technique: a 10-year experience in 100 patients, J. Craniofac. Surg. 14 (2003) 521-525.

[6] L. Caouette-Laberge, N. Guay, P. Bortoluzzi, C. Belleville, Otoplasty: anterior scoring technique and results in 500 cases, Plast. Reconstr. Surg. 105 (2000) $504-515$.

[7] A. de la Fuente, G. Sordo, Minimally invasive otoplasty: technical details and long-term results, Aesthetic Plast.Surg 36 (2012) 77-82.

[8] A.K. Gosain, A. Kumar, G. Huang, Prominent ears in children younger than 4 years of age: what is the appropriate timing for otoplasty? Plast. Reconstr. Surg. 114 (2004) 1042-1054.

[9] C.F. Haas, A. Champion, D. Secor, Motivating factors for seeking cosmetic surgery: a synthesis of the literature, Plast. Surg. Nurs. 28 (2008) 177-182.

[10] P.I. Heidekrueger, S. Juran, D. Ehrl, T. Aung, N. Tanna, P.N. Broer, Global aesthetic surgery statistics: a closer look, J.Plast.Surg. Hand Surg. (2016) 1-5.

[11] S. Irkoren, D. Kucukkaya, N. Sivrioglu, H.S. Ozkan, Using bilaterally fascioperichondrial flaps with a distal and a proximal base combined with conventional otoplasty, Eur. Arch. Otorhinolaryngol. 271 (2014) 1389-1393.

[12] P. Kelley, L. Hollier, S. Stal, Otoplasty: evaluation, technique, and review, J. Craniofac. Surg. 14 (2003) 643-653.

[13] A. Mandal, H. Bahia, T. Ahmad, K.J. Stewart, Comparison of cartilage scoring and cartilage sparing otoplasty-A study of 203 cases, J. Plast. Reconstr. Aesthet. Surg. 59 (2006) 1170-1176.

[14] P. Maricevich, N.F. Gontijo de Amorim, R. Duprat, F. Freitas, I. Pitanguy, Island technique for prominent ears: an update of the Ivo Pitanguy clinic experience, Aesthet. Surg. J. 31 (2011) 623-633.

[15] B. Olivier, H. Mohammad, A. Christian, R. Akram, Retrospective study of the long-term results of otoplasty using a modified Mustarde (cartilage-sparing) technique, J. Otolaryngol. Head. Neck. Surg. 38 (2009) 340-347.

[16] S.S. Pawar, C.A. Koch, C. Murakami, Treatment of prominent ears and otoplasty: a contemporary review, JAMA Facial Plast.Surg 17 (2015) 449-454.

[17] F. Peker, B. Celikoz, Otoplasty: anterior scoring and posterior rolling technique in adults, Aesthetic Plast.Surg 26 (2002) 267-273.

[18] H. Raunig, N. Kang, Correction of the hypertrophic conchal bowl without cartilage excision, J. Plast. Reconstr. Aesthet. Surg. 67 (2014) 1393-1396.

[19] J.A. Ribeiro, G.S. da Silva, Finesse in otoplasty in four steps, Aesthetic Plast.Surg 36 (2012) 846-852.

[20] M. Salgarello, C. Gasperoni, A. Montagnese, E. Farallo, Otoplasty for prominent ears: a versatile combined technique to master the shape of the ear, Otolaryngol. Head. Neck. Surg. 137 (2007) 224-227.

[21] C. Schlegel-Wagner, G. Pabst, W. Muller, T. Linder, Otoplasty using a modified anterior scoring technique: standardized measurements of long-term results, Arch. Facial Plast.Surg 12 (2010) 143-148.

[22] M. Sinha, B. Richard, Postauricular fascial flap and suture otoplasty: a prospective outcome study of 227 patients, J. Plast. Reconstr. Aesthet. Surg. 65 (2012) 367-371.

[23] M. Songu, A. Kutlu, Long-term psychosocial impact of otoplasty performed on children with prominent ears, J. Laryngol. Otol. 128 (2014) 768-771.

[24] F.J. Stucker, N.M. Vora, T.S. Lian, Otoplasty: an analysis of technique over a $33-$ year period, Laryngoscope 113 (2003) 952-956.

[25] L.F. Ungarelli, C.Z. de Andrade, E.G. Marques, J.L. Jorge, B.F. Neto, G.A. de Andrade, et al., Diagnosis and prevalence of prominent lobules in otoplasty: analysis of 120 patients with prominent ears, Aesthetic Plast.Surg 40 (2016) 645-651.

[26] F. Werdin, M. Wolters, H. Lampe, Pitanguy's otoplasty: report of 551 operations, Scand.J.Plast.Reconstr.Surg. Hand Surg. 41 (2007) 283-287.

[27] P. Yugueros, J.A. Friedland, Otoplasty: the experience of 100 consecutive patients, Plast. Reconstr. Surg. 108 (2001) 1052-1053, 1045-51; discussion. 